\title{
Qualitative analysis of irregular fields delivered with dual electron multileaf collimator: A Monte Carlo study
}

\author{
Samuel Okon Inyang ${ }^{1,2, a}$, Alan Chamberlain ${ }^{1}$ \\ ${ }^{I}$ Department of Medical Physics, University of Limpopo, Medunsa Campus, Medunsa 0204, South Africa \\ ${ }^{2}$ Department of Physics, University of Calabar, Calabar, Nigeria \\ ${ }^{a}$ E-mail address: inyangso@yahoo.com
}

\begin{abstract}
The use of a dual electron multileaf collimator (eMLC) to collimate therapeutic electron beam without the use of cutouts has been previously shown to be feasible. Further Monte Carlo simulations were performed in this study to verify the nature and appearance of the isodose distribution in water phantom of irregular electron beams delivered by the eMLC. Electron fields used in this study were selected to reflect those used in electron beam therapy. Results of this study show that the isodose distribution in a water phantom obtained from the simulation of irregular electron beams through the eMLC conforms to the pattern of the eMLC used in the delivery of the beam. It is therefore concluded that the dual eMLC could deliver isodose distributions reflecting the pattern of the eMLC field that was used in the delivery of the beam.
\end{abstract}

Key words: irregular fields; Monte Carlo; electron multileaf collimator; cut-out; applicator.

\section{Introduction}

Electron therapy is preferred in the treatment of superficial tumours due to the inherent advantage of high energy electrons obtained from medical linear accelerators (LINAC) to produce a sharp drop off in dose beyond the tumour tissue. Electron therapy is useful in the treatment of head, neck, skin and lip cancers, as well as in chest wall irradiation applied in breast cancer and boost dose to nodes. Khan [1] maintains that electron beam therapy is superior in some cases of radiation treatment as it is able to produce homogeneous dose in the target. Proper shaping of electron field is important to avoid undue exposure of healthy tissues to high doses. For proper protection of healthy tissues, radiation treatment is conducted after completion of appropriate treatment planning and the demarcation of tumour margins. This process ensures that the tumour receives a homogeneous radiation dose while healthy tissues and critical organs are protected. It is worthy to note that the treatment planning should be able to provide reproducible set-ups [2].

Presently, electron field shaping is performed with the use of applicators and cut-outs. The use of electron multileaf collimators (eMLC) is still being investigated and has not yet been standardized for everyday clinical use [3-9]. The effect of field shaping in electron therapy is important especially when irregular fields are used; as they have been found to affect dose output in a complex manner [1,10-11].

Although an eMLC can be used to deliver irregular electron field for therapy, there are not sufficient data to allow for its routine clinical use. The present study is set up to collect dosimetry data on a dual eMLC that could deliver irregular electron fields, without the presence of applicators and cutouts.

This study was carried out by simulating the eMLC designed by Inyang and Chamberlain [12,13] using the EGSnrc Monte Carlo (MC) code. Electrons passing through the linear accelerator head (LINAC) and shaped by the dual eMLC to produce irregular fields were used to produce the dose distributions in a water phantom. Several investigators [14,15] have shown that Monte Carlo calculation of dose distributions is the most accurate and reliable as compared to other methods. This study was therefore designed to verify the shape of the isodoses in water phantom obtained with the use of irregular electron beams shaped with the dual eMLC. The dose distributions were calculated using the EGSnrc Monte Carlo code.

\section{Materials and Methods}

The design of the dual eMLC and the comparison of its dosimetric parameters with measured values obtained by use of Varian type III applicators were presented in previous works $[12,13]$. Methods described previously were used to set the upper and lower eMLCs relative to each other as well as to the other component modules in the accelerator [12].

A summary of the Monte Carlo simulation of the eMLC is given in this study to ensure its completeness. The eMLC consists of the upper and lower eMLCs with leaves' width and thickness that allow for a maximum field size of $20 \times 20 \mathrm{~cm}^{2}$ at the distance of $100 \mathrm{~cm}$ from source to surface (SSD). The 
Monte Carlo simulations were done using the BEAMnrc and DOSXYZnrc which are user codes of EGSnrc used for modelling radiation transport through the linac treatment head and calculation of dose distributions in water phantom respectively.

Input into the EGSnrc code was based on the information supplied by Varian Incorporated concerning the medical linear accelerator head components on a non disclosure agreement with the authors. The PRESTA algorithm and other EGSnrc parameters used in this study were set at default values which have been established to be adequate for linac simulations [16]. The electron cut off (ECUTIN) energy and photon cut off (PCUTIN) energy were set to $0.521 \mathrm{MeV}$ and $0.010 \mathrm{MeV}$ respectively while the simulation time (TIMMAX) required to prevent the simulation from being aborted due to insufficient time was set to 900 hours. Details of input and output analysis of EGSnrc simulations are recorded in Walters and Rogers [17].

EGSnrc Monte Carlo code is designed such that the central beam axis corresponds to the $\mathrm{z}$-axis of the Cartesian coordinates; with all component modules of the accelerator arranged perpendicular to the $\mathrm{z}$-axis in the xy-plane.

In this study, irregular fields mean non-rectangular and noncircular fields. Attention was given to fields of sizes equivalent to $10 \times 10 \mathrm{~cm}^{2}$ or smaller, not exceeding the maximum field possible with the eMLC. Four irregular fields were arbitrarily selected, though with the intention to reflect those commonly used in the clinic for treatments. The nature of the fields and the isodose distributions obtained from these fields are given in the results section. After the simulation of these fields with BEAMnrc and dose calculations with DOSXYZnrc codes, the isodose distributions within and around the fields were investigated using dosxyz_show, a software package for displaying dose distributions which are regarded as the best illustration of the collimation effect of the dual eMLC.

All isodose distributions considered in this study were analysed around the beam central axis at depth of maximum dose which is regarded as the most sensitive depth for the assessment of beam homogeneity parameters [18]. The isodose curves within the isodose distributions are values of absorbed dose expressed as a percentage of the maximum dose along the beam central axis. The irregular fields used in this study are indicated as IRR1-4 and shown in figures 1-5.

\section{Results}

Previously, it was established that the electron beams delivered by the eMLC system were symmetrical and flat at depth of the maximum dose [12]. The simulations of the dose distributions for fields shaped by the dual eMLC, represented by the isodoses calculated at depth of maximum dose, were compared with the field patterns formed by the eMLC. Results of these simulations are presented in figures 1-4. All isodose curves presented in figures 1-4 were calculated in the $\mathrm{x}-\mathrm{y}$ plane.

In figure $\mathbf{5}$ the dose distribution for irregular fields (IRR1) is presented. The dose distributions for other irregular fields (IRR2, IRR3 and IRR4) are similar. They are not presented in this paper.
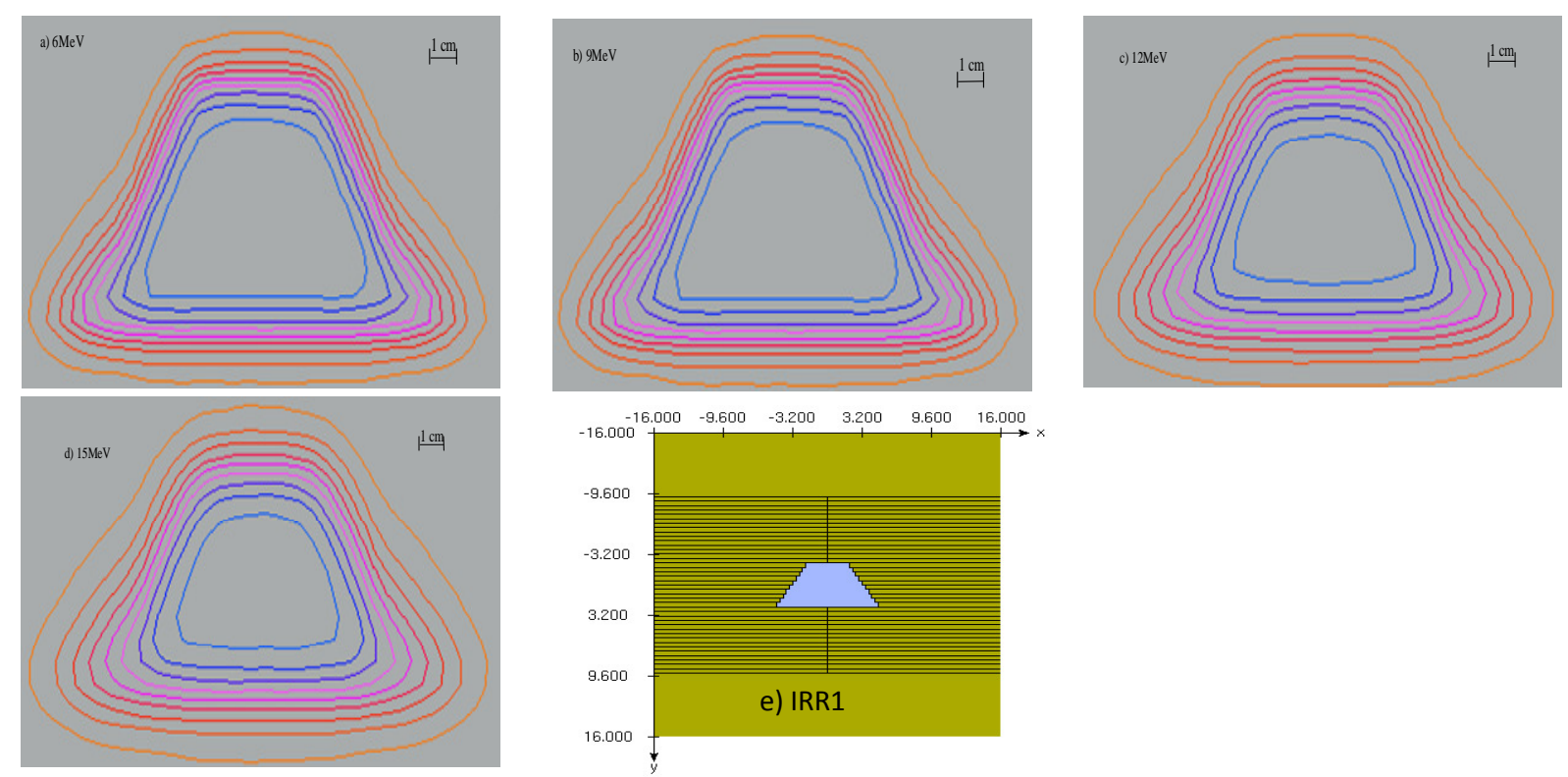

Figure 1. Irregular field (IRR1) with the associated simulated isodose curves in $x-y$ plane for the different energies starting inside with the $90 \%$ isodose line and descending in steps of $10 \%$ with $\pm 1.2 \%$ uncertainty and isodose shift of about $0.2 \mathrm{~cm}$. 

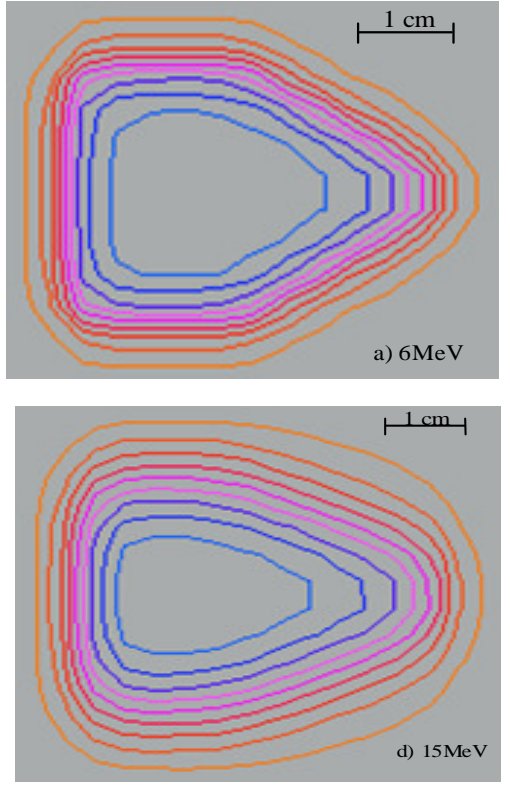
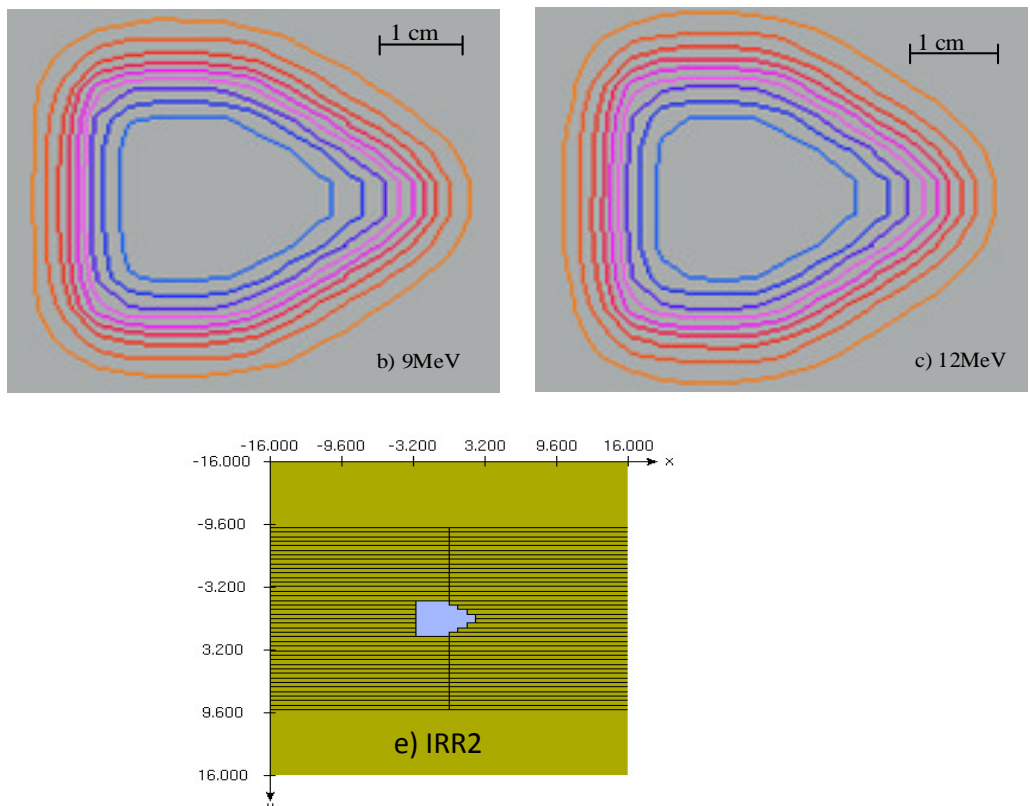

Figure 2. Irregular field (IRR2) with the associated simulated isodose curves in $x-y$ plane for the different energies starting inside with the $90 \%$ isodose line and descending in steps of $10 \%$ with $\pm 1.2 \%$ uncertainty and isodose shift of about $0.2 \mathrm{~cm}$.
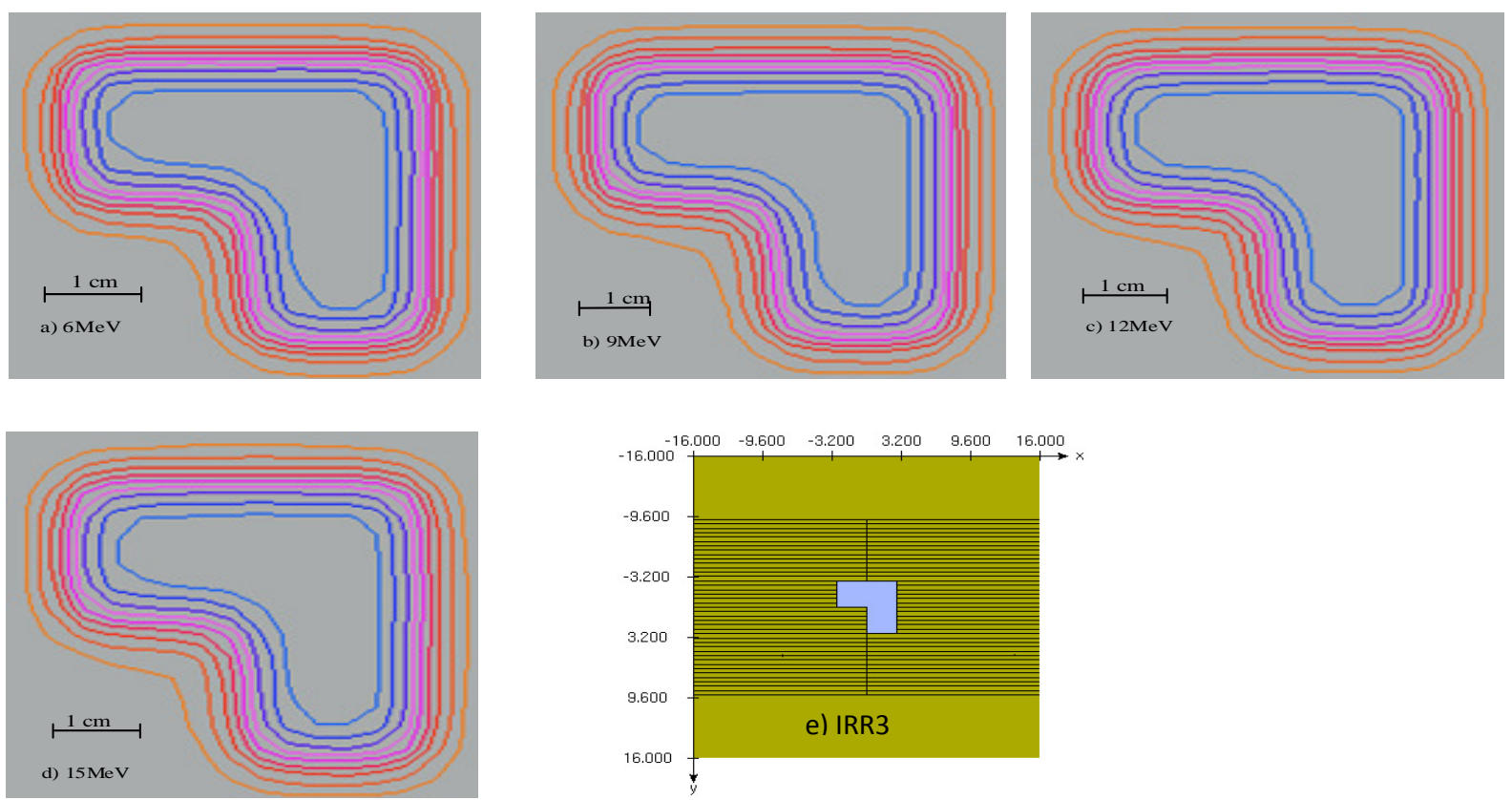

Figure 3. Irregular field (IRR3) with the associated simulated isodose curves in $x-y$ plane for the different energies starting inside with the $90 \%$ isodose line and descending in steps of $10 \%$ with $\pm 1.2 \%$ uncertainty and isodose shift of about $0.2 \mathrm{~cm}$.
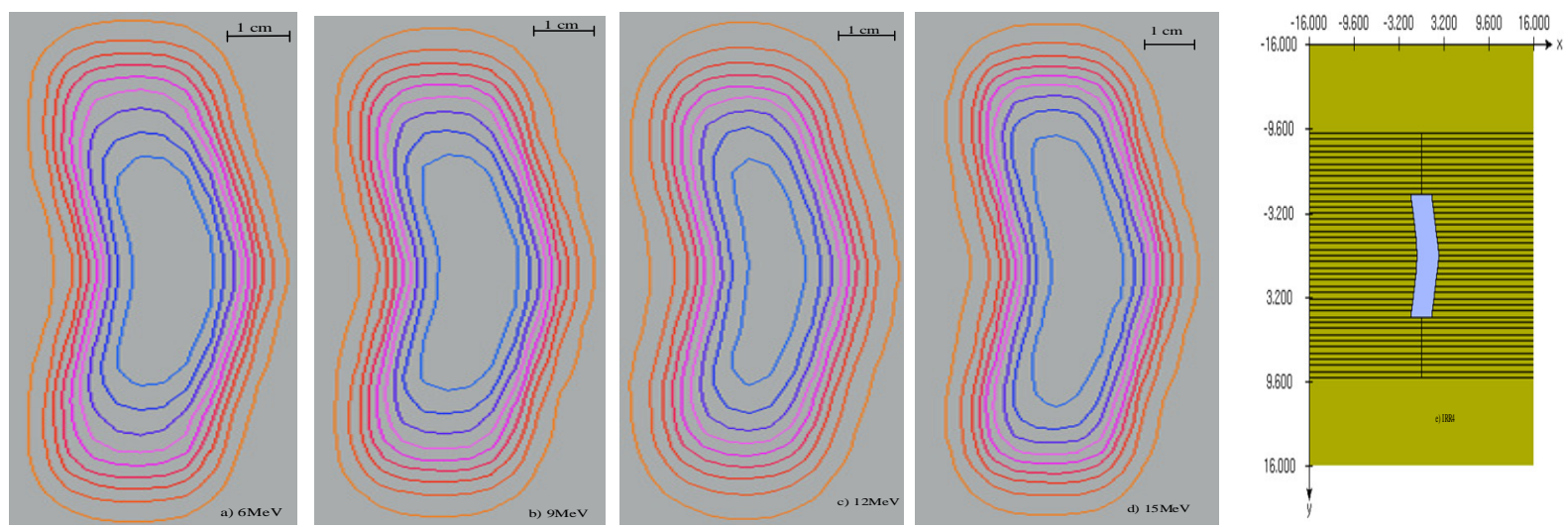

Figure 4. Irregular field (IRR4) with the associated simulated isodose curves in $x-y$ plane for the different energies starting inside with the $90 \%$ isodose line and descending in steps of $10 \%$ with $\pm 1.2 \%$ uncertainty and isodose shift of about $0.2 \mathrm{~cm}$. 

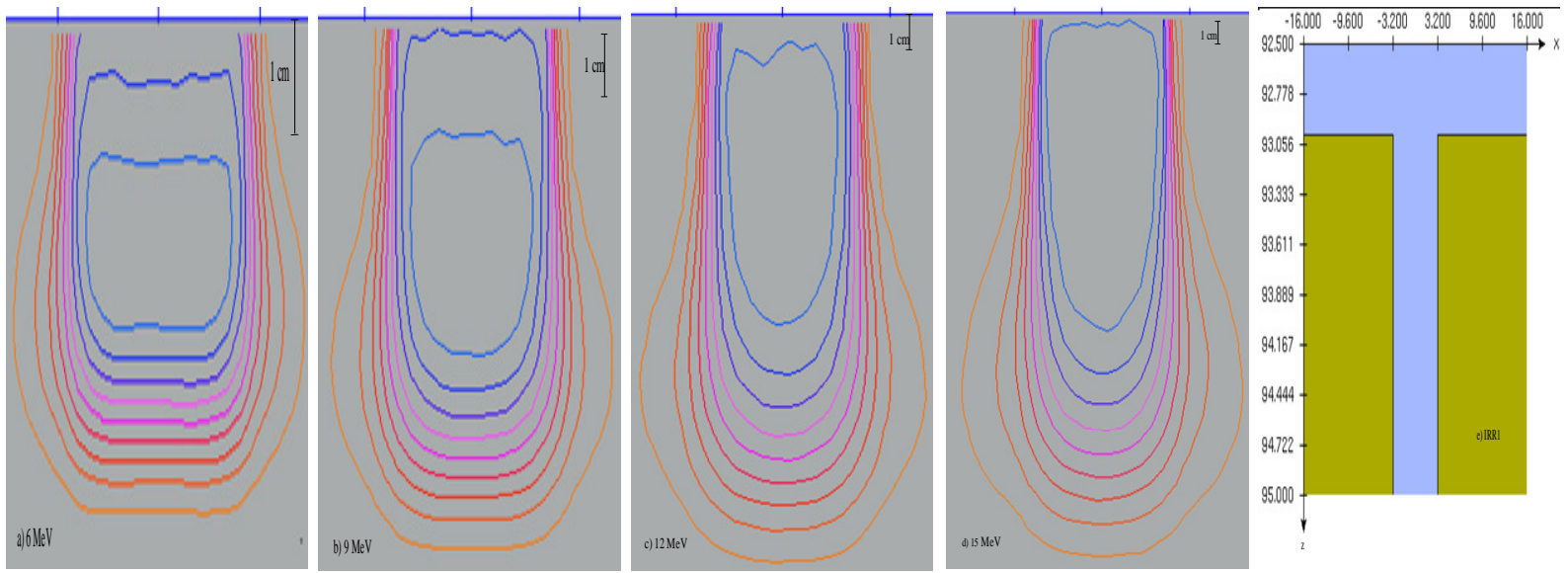

Figure 5. Irregular field (IRR1) with the associated isodose curves in $x-z$ plane for the different energies starting inside with the $90 \%$ isodose line and descending in steps of $10 \%$ with $\pm 1.2 \%$ uncertainty and isodose shift of about $0.2 \mathrm{~cm}$.

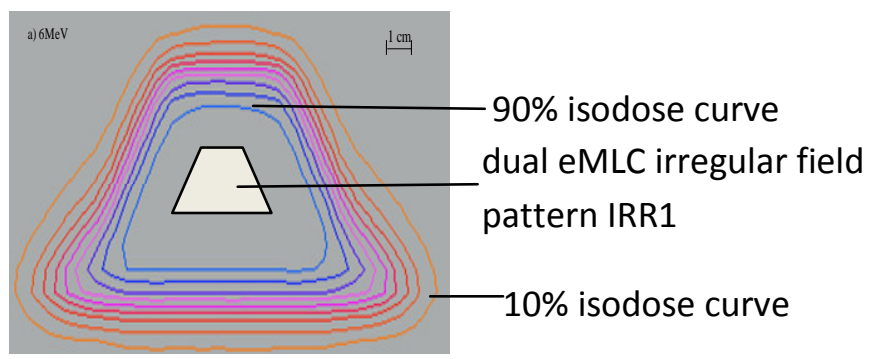

Figure 6. eMLC irregular field pattern IRR1 embedded in the centre of isodose distribution formed by the field.

\section{Discussion}

The isodose curves of the different irregular fields and the corresponding field patterns are given in figures 1-4 to show the dose distributions in phantom as delivered by the different irregular beams from the dual eMLCs. In all cases, it is observed that the isodose curves are arranged in a manner that map out the pattern of the dual eMLC irregual fields that produced them. Figure 6 contains the irregular field pattern (IRR1) embedded in one of the isodose distributions in Figure 1 and clearly illustrates that the isodose distributions conform to the field that generated them. Similar illustrations for other field patterns are not shown here in order to save journal space. However, they all display similar patern as shown in figure 6. It is therefore possible to state that the eMLC was able to resolve the fields and maintain the original leaf pattern in the dose distribution in the phantom.

Figure 5, which indicates the penetration of the irregular beams as obtained from irregular field 1 (IRR1), shows the
$90 \%$ isodose level of $6 \mathrm{MeV}, 9 \mathrm{MeV}, 12 \mathrm{MeV}$ and $15 \mathrm{MeV}$ as the innermost isodose line. The $90 \%$ isodose levels have depth penetration of $2.0 \mathrm{~cm}, 3.0 \mathrm{~cm}, 3.7 \mathrm{~cm}$ and $4.5 \mathrm{~cm}$ at $6 \mathrm{MeV}$, $9 \mathrm{MeV}, 12 \mathrm{MeV}$ and $15 \mathrm{MeV}$ respectively. It is recommended that the $90 \%$ isodose level should enclose the planning target volume to enhance the delivery of the required dose to the tumour site [14]. The $80 \%$ isodose level can enclose a target lying within the depths of $2.2 \mathrm{~cm}, 3.5 \mathrm{~cm}, 4.4 \mathrm{~cm}$ and $5.0 \mathrm{~cm}$ at $6 \mathrm{MeV}, 9 \mathrm{MeV}, 12 \mathrm{MeV}$ and $15 \mathrm{MeV}$ respectively. Du Plessis et al [15] demonstrated target coverage within the $70 \%$ isodose contour using the irregular fields of photon multileaf collimator (pMLC). If this is applied in the present situation, the target will be encompassed within $2.4 \mathrm{~cm}, 3.4 \mathrm{~cm}, 5.0 \mathrm{~cm}$ and $5.8 \mathrm{~cm}$ at $6 \mathrm{MeV}, 9 \mathrm{MeV}, 12 \mathrm{MeV}$ and $15 \mathrm{MeV}$ respectively.

The $20 \%$ isodose levels were at depths of $3.0 \mathrm{~cm}, 4.5 \mathrm{~cm}$, $6.1 \mathrm{~cm}$ and $7.5 \mathrm{~cm}$ for $6 \mathrm{MeV}, 9 \mathrm{MeV}, 12 \mathrm{MeV}$ and $15 \mathrm{MeV}$, respectively. These depths are close to the $R_{20}$ value of the eMLC's for $10 \times 10 \mathrm{~cm}^{2}$ field - the $2.9 \mathrm{~cm}, 4.3 \mathrm{~cm}, 6.1 \mathrm{~cm}$ and $7.5 \mathrm{~cm}$ for $6 \mathrm{MeV}, 9 \mathrm{MeV}, 12 \mathrm{MeV}$ and $15 \mathrm{MeV}$, respectively.

\section{Conclusions}

The isodose curves produced by the different irregular electron fields formed by the dual eMLC in this study reflect the field pattern specified by the eMLC. The dual eMLC is capable to form isodose curves that have similar pattern to the fields shaped by the cut-outs.

\section{References}

[1] Khan FM. The physics of radiation therapy. 3rd edition. Philadelphia: Lippincott Williams and Wilkins; 2003.

[2] Stanton R, Stinson D. Applied radiation physics for radiation oncology. Madison (Wisconsin): Medical Physics Publishing; 1996.

[3] Lee MC, Jiang SB, Ma C-M. Monte Carlo and experimental investigation of multileaf collimated electron beams for modulated electron radiation therapy. Med Phys. 2000;27(12):2708-2718.

[4] Ma C-M, Pawlicki T, Lee MC, et al. Energy- and intensity- modulated electron beams for radiotherapy. Phys Med Biol. 2000;45(8):2293-2311. 
[5] Ravindran BP, Singh IR, Brindha S, et al. Manual multi-leaf collimator for electron beam shaping - a feasibility study. Phys Med Biol. 2002;47(24):4389-4396.

[6] Blomquist M, Karlsson MG, Zackrisson B, et al. Multileaf collimation of electrons - clinical effects on electron energy modulation and mixed beam therapy depending on treatment head design. Phys Med Biol. 2002;47(7):1013-1024.

[7] Karlsson MG, Karlsson M. Electron collimation with focused and curved leaf end MLCs - experimental verification of Monte Carlo optimized designs. Med Phys. 2002;29(4):631-637.

[8] Hogstrom KR, Boyd RA, Antolak JA, et al. Prototype retractable eMLC for fixed-beam electron therapy. Phys Med Biol. 2004;31(3):443-462.

[9] Olofsson L, Karlsson MG, Karlson M. Effects on electron penumbra using the photon MLC to reduce bremsstrahlung leakage from add-on electron MLC. Phys Med Biol. 2005;50(6):1191-1203.

[10] McParland BJ. A method of calculating the output factors of arbitrarily shaped electron fields. Med Phys. 1989;16(1):88-93.

[11] Washington CM, Leaver DT. Principles and practice of radiation therapy: Physics, simulation, and treatment planning. St Louis (Missouri): Mosby; 1996.

[12] Inyang SO, Chamberlain AC. Monte Carlo simulations of electron beams collimated with dual electron multileaf collimator: a feasibility study. Radiol Phys Tech. 2009;2(2):210-218.

[13] Inyang SO, Chamberlain AC. Optimization of the dual electron multileaf collimator materials using the EGSnrc. Radiol Phys. Tech. 2010;3(2):165-170.

[14] Kudchadker RJ, Hogstrom KR, Garden AS, et al. Electron conformal radiotherapy using bolus and intensity modulation. Int Journal Radiat Oncol Biol Phys. 2002;53(4):1023-1037.

[15] Du Plessis FC, Leal A, Stathakis S, et al. Characterization of megavoltage electron beams delivered through multi-leaf collimator (pMLC). Phys Med Biol. 2006;51(8):2113-2129.

[16] Rogers DWO, Ma C-M, Walters B, et al. BEAMnrc Users Manual. Ottawa: National Research Council (NRC) of Canada, Report PIRS-0509(A): 2002.

[17] Walters BRB, Rogers DWO. DOSXYZnrc Users Manual. Ottawa: National Research Council (NRC) of Canada, Report PIRS-794: 2002.

[18] IAEA. Review of Radiation Oncology Physics: A Handbook for Teachers and Students. Vienna: International Atomic Energy Agency, Educational Report Series: 2003. 